\title{
Novel Reconstruction Using Roux-en-Y Plus Antral Obstruction Following Proximal Gastrectomy: Feasibility and Quality of Life Benefits
}

Dejun Yang ( $\nabla$ yangdejun_smmu@163.com )

Changzheng Hospital https://orcid.org/0000-0003-0090-0298

Changming Wang

Changzheng Hospital

Hongbing Fu

Changzheng Hospital

Ziran Wei

Changzheng Hospital

Xin Zhang

Changzheng Hospital

Zunqi Hu

Changzheng Hospital

Yu Zhang

Changzheng Hospital

Qingping Cai

Changzheng Hospital

\section{Research article}

Keywords: proximal gastrectomy; gastroesophagostomy; digestive tract reconstruction; Roux-en-Y anastomosis; double tract reconstruction; bile reflux gastritis

Posted Date: July 16th, 2019

DOI: https://doi.org/10.21203/rs.2.11422/v1

License: (c) (1) This work is licensed under a Creative Commons Attribution 4.0 International License.

Read Full License 


\section{Abstract}

Background and Aims Routine gastroesophagostomy has been shown to have adverse effects on the recovery of digestive functions and quality of life because patients typically experience reflux symptoms after proximal gastrectomy. This study was performed to assess the feasibility and quality of life benefits of a novel reconstruction method termed Roux-en-Y anastomosis plus antral obstruction (RYAO) following proximal partial gastrectomy. Methods A total of 73 patients who underwent proximal gastrectomy from June 2015 to June 2017 were divided into two groups according to digestive reconstruction methods [RYAO (37 patients) and conventional esophagogastric anastomosis with pyloroplasty (EGPP, 36 patients)]. Clinical data were compared between the two groups retrospectively. Results The mean operative time for digestive reconstruction was slightly longer in the RYAO group than in the EGPP group. However, the incidence of postoperative short-term complications did not differ between the RYAO and the EGPP groups. At the 6-month follow-up, the incidence rates of both reflux esophagitis and gastritis were lower in the RYAO group than in the EGPP group $(P=0.002)$. Additionally, body weight recovery was better in the RYAO group $(P=0.028)$. The scale tests indicated that compared with the patients in the EGPP group, the patients in the RYAO group had significantly reduced reflux, nausea and vomiting and reported improvements in their overall health status and quality of life (all $\mathrm{P}<$ 0.05). Conclusion RYAO reconstruction may be a feasible procedure to reduce postoperative reflux symptoms and the incidence of reflux esophagitis and gastritis, thus improving patient quality of life after proximal gastrectomy.

\section{Abstract}

Background Conventional gastroesophagostomy with pyloroplasty (EGPP) have adverse effects on postoperative recovery because of reflux symptoms after proximal gastrectomy, moreover, double tract reconstruction (DT) is also not the ideal procedure due to the complexity of operation. This study was performed to assess a novel reconstruction method termed Roux-en-Y anastomosis plus antral obstruction (RYAO) followed proximal partial gastrectomy.

Methods A total of 104 patients who underwent proximal gastrectomy from June 2015 to June 2017 were divided into three groups according to digestive reconstructions [RYAO (37 patients), EGPP (36 patients) and DT (31 patients)]. Clinical data were compared between the groups retrospectively.

Results Both operative duration and reconstruction time were significantly longer in the DT group than in the EGPP group and RYAO group, so was the hospital stay. The incidence of postoperative short-term complications did not differ between the three groups. At the 6-month follow-up, the incidence rates of bile reflux gastritis were lower in the RYAO group than in the EGPP group and DT group. Additionally, body weight recovery was better in the RYAO group. The scale tests indicated that compared with the EGPP group and DT group, the patients in the RYAO group had significantly reduced reflux symptoms, and reported improvements in their overall health status and quality of life. 
Conclusions RYAO reconstruction may be a feasible procedure to reduce postoperative reflux symptoms and the incidence of reflux gastritis and esophagitis, thus improving patient quality of life after proximal gastrectomy.

Keywords proximal gastrectomy; gastroesophagostomy; digestive tract reconstruction; Roux-en-Y anastomosis; double tract reconstruction; bile reflux gastritis

\section{Background}

The incidence of precancerous or borderline lesions in the esophagogastric junction (EGJ), including adenomatoid polyps, leiomyomas, stromal tumors and ulcers with dysplasia, is relatively high, and some of these conditions require surgical treatment [1-5]. Proximal gastrectomy is a widely used surgical method for these diseases that can achieve complete resection of lesions. The range of resection is usually less than $1 / 2$ of the stomach volume. The remaining stomach capacity is sufficient to maintain and restore digestive function [6-8].

Gastrointestinal reconstruction is the final and key step of gastrectomy, including proximal gastrectomy. This procedure directly affects the recovery of digestive functions and patients' nutritional status and quality of life after surgery [9-11]. The most commonly used reconstruction method after proximal gastrectomy is esophagogastric anastomosis. Reflux of digestive fluid and food into the esophagus is common due to the relatively weak emptying function of the residual stomach. Pyloroplasty, which is performed to prevent postoperative gastric retention, can cause digestive fluid in the duodenum to retrograde flow into the stomach and then the esophagus, resulting in reflux gastritis, reflux esophagitis and anastomotic inflammation. This condition presents with symptoms such as an abdominal burning sensation, belching, acid reflux, and nausea and vomiting, which seriously affect postoperative diet and quality of life, thereby adversely affecting the physical and mental health of these patients. Some even suffer from depression, anxiety and malnutrition [12-14].

To overcome the abovementioned shortcomings of gastroesophagostomy, surgeons had performed some antireflux procedures such as total gastrectomy followed by Roux-en-Y (RNY) esophagojejunostomy, Merendino procedure (resection of the gastroesophageal junction with jejunal interposition), and double tract reconstruction which was commonly used, however, each of these procedures had its own limitations concerning on both antireflux and retain good digestive function, moreover, the complexity of the operations had been increased[15-17]. For above reasons, our research group designed a novel approach combining RNY anastomosis with antral obstruction (RYAO) for digestive tract reconstruction $[18,19]$. Since June 2015 , this novel approach has been applied to patients who have undergone proximal gastrectomy and met the surgical indications. In this article, we describe the procedural details of RYAO. In addition, the short-term safety and long-term benefits for quality of life were evaluated by comparing the novel approach with routine esophagogastric anastomosis (EGPP) and double tract reconstruction (DT). 


\section{Patients And Methods}

\section{Patients}

From June 2015 to June 2017, a total of 37 patients with lesions in the EGJ underwent RYAO in our center. Clinicopathological features, surgical data, perioperative and long-term complications and postoperative follow-up data of the patients who underwent RYAO were collected and compared with those of 36 patients who underwent EGPP and 31 patients who underwent DT during the same period. Preoperative assessments included gastroscopy and biopsy and, if necessary, endoscopic ultrasonography, contrast-enhanced computed tomography (CT), and other routine examinations.

The inclusion criteria for patients were as follows: (1) the lesion was located at the EGJ; (2) surgery was indicated for these lesions, which were not suitable for endoscopic therapy after discussion with the endoscopist; (3) the lesions were precancerous or borderline lesions confirmed by pathology or lesions of an unknown nature preoperatively; (4) the resection range of proximal gastrectomy was $\leq 1 / 2$ of the stomach volume, and the surgical margin met the criteria of the guidelines; (5) there were no surgical contraindications, and (6) the patients had no history of previous surgery.

The Ethics Committee of the Second Military Medical University had approved this study. Patients and their family members were informed about the details of each specific surgical procedure (RYAO or EGPP or DT) and were educated about the advantages and disadvantages of each surgical approach before they made any decisions. The patients and their family members provided consent and signed the informed consent form before surgery.

\section{Surgical procedure for proximal gastrectomy with RYAO}

If no ascites, adhesions and other abnormal lesions were noted in the abdominal cavity and the regional lesion could be surgically resected, proximal gastrectomy was carried out first, as described below.

The perigastric vessels were divided with preservation of the distal gastric artery and its branches to the gastric wall, including the right gastroepiploic artery and right gastric artery (Fig. 1A, B). The left gastroepiploic artery and left gastric artery as well as other gastric vessels could be excised. The esophagus was transected $2-3 \mathrm{~cm}$ above the gastric cardia, and an anvil was inserted into the esophageal lumen. A 75-mm linear cutter (NTLC75, Ethicon Endo-Surgery, OH, USA) was used to incise the gastric corpus and close the gastric stump in the greater curvature (Fig. 1C, D). Another 75-mm linear cutter was used to incise and close the gastric body in the lesser curvature; then, the residual gastric tube was formed (Fig. 1E, F).

Next, digestive tract reconstruction was performed in the following order: esophagogastric anastomosis, obstruction of the antrum and RNY anastomosis. 
At the lowest point in the greater curvature of the stomach, a 3-cm incision was made in the anterior wall of the gastric body. A circular stapler was inserted into the residual stomach. The trocar of the circular stapler was advanced through the posterior wall (approximately $3 \mathrm{~cm}$ distal to the stump) and attached to the anvil for side-end anastomosis of the esophagus and stomach (Fig. 2A, B) to complete the esophagogastric anastomosis. The pylorus was located, and the omentum from the vascular arch to the greater and lesser curvatures of the gastric antrum were separated. At a site approximately $2-3 \mathrm{~cm}$ from both the pylorus and the previous gastric incision, a 60-mm stapler (XF-60, XINNENGYUAN, Changzhou City, China) was used to close but not cut off the antrum (Fig. 2C, D). If rupture of the stomach wall did not occur and no bleeding from the wound was noted, then the obstruction of the antrum was considered complete (Fig. 2E, F). The jejunum was divided at a site $15 \mathrm{~cm}$ distal to the ligament of Treitz. Gastrojejunal side-side anastomosis was completed with hand-sewn sutures between the efferent loop incision and the previous incision in the residual stomach wall (Fig. 2G, H). Then, the stump of the afferent loop was anastomosed to the efferent loop $40 \mathrm{~cm}$ distal to the gastrojejunal anastomosis. Finally, the RNY anastomosis was completed.

After completion of RYAO, the novel reconstructed digestive tract included an esophageal-gastric-jejunal tract and a partially effective gastric antrum-duodenal tract with an obstructed proximal antrum (Fig. 3A, B).

\section{Routine esophagogastric anastomosis with pyloroplasty}

The pylorus was located, and a 2-cm incision was made in the anterior wall of the gastric pylorus along the longitudinal axis of the stomach. A circular stapler was inserted into the residual stomach. The trocar of the circular stapler was advanced through the posterior wall and attached to the anvil in the esophagus to complete the esophagogastric anastomosis. Then, the primary incision was hand-sutured vertical to the longitudinal axis of the stomach.

\section{Double tract reconstrucion with jejunal interposition}

The anvil of the circular stapler was inserted into the esophageal stump. The jejunum was divided $15 \mathrm{~cm}$ distal to the ligament of Treitz. An end-to-side jejunojejunostomy was created by an anastomosis between the $30 \mathrm{~cm}$ of anal jejunum from the oral jejunal stump and divided oral jejunum. An entry hole for the circular stapler was made halfway $(15 \mathrm{~cm})$ along the anal jejunal stump, and the circular stapler was used to achieve an end-to-side esophagojejunostomy. After removing the circular stapler, the anastomosis between the entry hole and the oral edge of the remnant stomach was made by hand sewing. The length of the jejunogastrostomy was $6 \mathrm{~cm}$.

\section{Postoperative management}


After surgery, routine gastrointestinal decompression was used. If no complications, including digestive tract leakage, hemorrhage or obstruction, were evident, then the gastric decompression tube was removed after gastrointestinal motility was restored and passage of flatus was reported. The patients were given an appropriate amount of water. One day later, a liquid diet was initiated if no discomfort was reported. Two days later, a semiliquid diet was initiated. If no discomfort was reported on the second day after starting the semiliquid diet and no abnormalities were noted in the drainage volume and color, then the abdominal drainage tube was removed, and the patient was discharged from the hospital.

\section{Prognostic evaluation}

Between June 2015 and June 2017, 37 patients underwent RYAO, 36 patients underwent EGPP, and 31 patients underwent DT. The clinicopathological features, the surgical procedure notes, perioperative complications, long-term complications, weight changes, health status and quality of life were documented and compared between the three reconstruction groups.

All 104 patients who underwent proximal gastrectomy were reevaluated at a 6-month postoperative follow-up examination, which included symptom assessment, physical examination, routine blood and liver and kidney function tests, endoscopy, iodine radiography of upper GI tract, abdominal ultrasonography and, gastrointestinal symptoms (EORTC QLQ-STO22 scores) [20, 21] and quality of life (EORTC QLQ-Core 30 scores) [22, 23].

Bile reflux gastritis was diagnosed by upper GI endoscopy and bilirubin level assessment of gastric aspirate. The diagnostic criteria was as follows: intragastric bile and residual gastritis was found under endoscopy, bilirubin level was exceeded normal serum range $(>1.3 \mathrm{mg} / \mathrm{dl})$. Reflux esophagitis were diagnosed by gastroscopy and iodine radiography of upper $\mathrm{GI}$ tract. The diagnostic criteria was as follows: endoscopically visible breaks in the distal esophageal mucosa, retrograde flow of gastrointestinal contents into the esophagus was detected by radiography.

\section{Statistical analysis}

All statistical analyses were performed with IBM SPSS Statistics for Windows, version 22.0 (Armonk, NY, USA). The Oneway ANOVA test was used to compare differences in normal distribution continuous variables between the groups, the Kruskal-Wallis test was used to compare differences in ordinal categorical and non-normal distribution continuous variables between the groups, and the Pearson ChiSquare test or Fisher's exact test was used to compare differences in unordered categorical variables between the groups. LSD-t test or Bonferroni test was used for pairwise comparison between groups, $P<$ 0.05 was considered statistically significant.

\section{Results}




\section{Clinical characteristics and surgical data of the patients}

The baseline demographics of the three groups of patients were comparable (Table 1). No organ dysfunction was reported in any of the patients, and no significant differences were observed in the lesion diameters or pathological types of tissue specimens between the three groups (Table 1).

No significant differences were observed in the intraoperative blood loss, time to first flatus and between the three groups. Both operative duration and reconstruction time were apparently longer in the DT group than in the EGPP group and the RYAO group, so was the postoperative hospital stay, while there was no significantly difference between the EGPP group and the RYAO group (Table 1).

\section{Postoperative complications, rehabilitation and quality of life}

The incidence of short-term complications was not significantly different between the three groups (Table 2). Moreover, intraabdominal bleeding, abdominal infections or abscesses, intestinal obstruction and organ dysfunction were not reported in the three groups of patients.

At the 6-month follow-up, the incidence of long-term complications was significantly lower in the RYAO group than in the EGPP group ( $13.5 \%$ vs. $44.4 \%$ ). Correspondingly, the incidence of bile reflux gastritis was significantly lower in the RYAO group than in the EGPP group (0/37 vs. 6/36). The incidence of reflux esophagitis was significantly lower in the RYAO group and the DT group than in the EGPP group (2/37 vs. $8 / 36,1 / 31$ vs. 8/36, respectively) (Table 2). The weight ratio (weight 6 months after surgery/weight before surgery) was $92.4 \pm 7.9 \%$ in the RYAO group and $86.8 \pm 9.8 \%$ in the EGPP group, suggesting that weight recovery was better in the RYAO group (Table 2). However, there was no difference between the RYAO group and the DT group in long-term complications, bile reflux gastritis, reflux esophagitis and weight ratio. No postoperative complications, including dumping syndrome, adhesive obstruction, cholecystitis and gallbladder stones, were reported in each group.

The QLQ-STO22 results suggested that the patients in the RYAO group had significantly fewer postoperative reflux symptoms than did those in the EGPP group and the DT group (Table 3). The QLQC30 scores showed that the patients in the RYAO group were less affected by the surgery and more comfortable performing daily activities (Role functioning) than were those in the EGPP group and the DT group. In addition, the symptom of nausea and vomiting was effectively relieved in the RYAO group compared to the EGPP group. Overall health status and quality of life improved significantly in the RYAO group compared to the EGPP group and the DT group (Table 4).

\section{Discussion}

Esophagogastric anastomosis after proximal gastrectomy was previously considered a simple procedure that preserved the storage function of the stomach and the physiology of the duodenal tract, supported food digestion and reduced the occurrence of malnutrition. However, the incidence of reflux was high due 
to the compromised lower esophageal sphincter (LES) and lack of the angle of His. Moreover, the compromised pyloric ring and contracture of the pyloric sphincter could easily lead to gastric emptying disorders and further aggravate reflux $[24,25]$.

To overcome these problems, sphincterotomy and pyloroplasty have become common practices following esophagogastric anastomosis and have reduced the incidence of gastric emptying dysfunction. Nevertheless, due to destruction of the cardiac and pyloric structures, reflux of not only bile into the stomach but also gastric contents into the esophagus occurs, and because the incidence of reflux is not significantly reduced, postoperative quality of life is not significantly improved [26-29]. Our findings are consistent with the above results, showing that patients with EGPP have notable reflux symptoms and a high incidence of reflux esophagitis and gastritis, which adversely affect their quality of life and health.

In view of another approach, the double tract reconstruction could reduce the incidence of reflux esophagitis and retained the gastric reservoir and duodenal passage, but the procedure was a little complicated and need more reconstruction time. Moreover, the volume function of residual stomach has not been fully developed because most of the ingested food did not pass through the gastric bypass, and the bile reflux gastritis had not been significantly reduced [30-33]. Similarly, our practices have showed that patients with DT also have apparently reflux symptoms and a relatively high incidence of bile reflux gastritis, which has a negative impact on their quality of life.

Therefore, developing methods to improve anastomosis following proximal gastrectomy has become a hot topic in the field of gastrointestinal surgery. The RYAO procedure has shown some advantages in preserving function and reducing reflux.

RYAO is an organized combination of esophagogastric anastomosis, antral obstruction (uncut blocking) and RNY gastrojejunostomy. Compared with EGPP, the RYAO procedure includes an additional RNY gastrojejunostomy step but has a reduced pyloroplasty step. In addition, the entire procedure does not require extra time because the short time needed for antral obstruction offsets the additional time required for gastrojejunostomy. Both RNY gastrojejunal anastomosis and antral blocking are relatively simple procedures that do not increase the difficulty of the surgery, rather, surgical safety and convenience could be ensured and the low incidence of perioperative symptoms were presented. Accordingly, the operative duration, the reconstruction time and postoperative hospital stay were significantly shorter in the RYAO group than in the DT group because of the differences of surgical complexity.

Sufficient gastric storage function is still ensured because of the retained more than $1 / 2$ of stomach in the RYAO group, and the preserved gastric body can secrete sufficient digestive fluid for digestion[34]. Although the gastric antrum is blocked, the digestive juice secreted by the gastric antrum distal to the block site can enter the duodenum, stimulate and maintain duodenal secretory function and partially preserve the duodenal physiological tract, moreover, facilitating restoration of the nerve-endocrine function of the digestive tract and promoting the release of gastrointestinal peptide hormones, including 
pancreozymin, cholecystokinin and insulin, which can enhance the digestion of food and thus reduce malnutrition. [35, 36].

In addition to maintaining digestive function, RYAO effectively prevents the occurrence of reflux. First, obstruction of the gastric antrum completely blocks the reflux of bile. Second, RNY gastrojejunostomy changes the drainage path of digestive fluid from horizontal to vertical. The effect of gravity and shortening of the path decrease the occurrence of gastric emptying dysfunction. Thus, as gastric emptying is promoted, gastroesophageal reflux is reduced.

Due to the sufficient capacity for food storage, the partially available physiological gastroduodenal tract and good digestive function, the incidence of postoperative malnutrition decreased, and weight recovery was relatively ideal. Furthermore, the incidence of reflux esophagitis and gastritis decreased, and the patients' reflux symptoms significantly improved. The patients experienced less discomfort during daily work and home activities, and their improved overall health status significantly enhanced their quality of life. Therefore, RYAO may be a promising technique for digestive tract reconstruction following proximal gastrectomy.

In contrast, several antireflux operations applied recently all had limitations. Despite the function of antireflux, total gastrectomy with RNY reconstruction had neither gastric reservoir nor duodenal passage that might add some post gastrectomy symptoms [37, 38]. Moreover, in view of the preservation of the duodenal passage and maintenance of the gastric reservoir, Merendino procedure might be an ideal anastomosis, however, the procedure was comlexity and high rate of postoperative complications was found $[15,16]$.

\section{Conclusions}

RYAO following proximal gastrectomy is feasible due to its simplicity and operational safety for patients with surgical indications. Moreover, RYAO reduces the occurrence of reflux while maintaining relatively good digestive function, and patients experience less discomfort and report improvements in their quality of life. In summary, RYAO may be a novel gastrointestinal reconstruction technique with practical significance.

Although the outcomes of this procedure are satisfactory, further research is needed to evaluate its efficacy and safety on a large sample by multicenter, randomized trial and to clarify the mechanism and the capacity of remnant stomach. In addition, extending the scope of the surgical indications and applications is another direction for future work.

\section{Declarations}

Ethics approval and consent to participate The study strictly follows the principle of fairness and justice and fully embodies the rights and interests of the subjects by respecting their rights to know as well as their rights of privacy. Furthermore, the study is in line with biomedical ethics research involving human 
subjects according to current Chinese policies and regulations and complies with the ethical standards proposed in the 1964 Declaration of Helsinki and its later amendments or comparable ethical standards. The study has been approved by the ethics committee of SMMU.

Consent for publication Not applicable

Availability of data and materials

The datasets used and analysed during the current study are available from the corresponding author on reasonable request.

Competing interests The authors declare that they have non-financial competing interests.

Funding This study was supported by the Science and Technology Commission of Shanghai Municipality (Grant No. 17411972200); the Natural Science Foundation of Shanghai (Grant No. 17ZR1439300); and the Scientific Research Program of Shanghai Municipal Commission of Health and Family Planning (Grant No. 201640269); the First Program Characterized by War Trauma of Changzheng Hospital (Grant No. 201711015); the Special Subject of Military Health Care (Grant No. 15BJZ26).

Authors' contributions

Dejun Yang wrote the paper. Dejun Yang and Changming Wang designed and improved the novel technique, interpreted the study data, and critically revised the manuscript for important intellectual contents. Hongbing Fu and Ziran Wei participated in the research and analyzed the data. Xin Zhang, Zunqi Hu and Yu Zhang collected the data. Qingping Cai designed and executed the novel reconstruction technique.

Acknowledgements Not applicable

\section{References}

1. Matsuda T, Takeuchi H. Clinicopathological Characteristics and Prognostic Factors of Patients with Siewert Type II Esophagogastric Junction Carcinoma: A Retrospective Multicenter Study: Reply. World J Surg. 2017;41:1395. 2. Diaconescu S, Miron I, Gimiga N, et al. Unusual Endoscopic Findings in Children: Esophageal and Gastric Polyps: Three Cases Report. Medicine (Baltimore). 2016;95:e2539. 3. Eckardt AJ, Lang $\mathrm{H}$, Gockel I. Diagnosis and therapy of benign tumors of the esophagogastric junction. Chirurg. 2014;85:1073-1080. 4. Jähne J. Benign diseases of the esophagogastric junction: A diagnostic and therapeutic challenge. Chirurg. 2014;85:1044-1045. 5. Morais DJ, Yamanaka A, Zeitune JM, et al. Gastric polyps: a retrospective analysis of 26,000 digestive endoscopies. Arq Gastroenterol. 2007;44:14-17. 6. Liao YT, Yang CY, Lai IR, et al. Laparoscopic resection for submucosal tumors near the esophagogastric junction: feasibility and short-term outcome. Surg Innov. 2013;20:478-483. 7. Levy RM, Trivedi D, Luketich JD. Minimally invasive esophagectomy. Surg Clin North Am. 2012;92:1265-1285. 8. Wiesel O, Whang B, Cohen D, et al. Minimally Invasive Esophagectomy for Adenocarcinomas of the Gastroesophageal 
Junction and Distal Esophagus: Notes on Technique. J Laparoendosc Adv Surg Tech A. 2017;27:162-169. 9. Motoyama K, Kojima K, Hayashi M, et al. $\beta$-Shaped intracorporeal Roux-en- $Y$ reconstruction after totally laparoscopic distal gastrectomy. Gastric Cancer. 2014;17:588-593. 10. Sasaki K, Miyachi K, Yoda N, et al. Long-term comparison of boomerang-shaped jejunal interposition and Billroth-I reconstruction after distal gastrectomy. World J Surg. 2015;39:1127-1133. 11. Gong JQ, Cao YK, Zhang GH, et al. Uncut Esophagojejunostomy with Double Jejunal Pouch: An Alternative Reconstruction Method that Improves the Quality of Life of Patients after Total Gastrectomy. J Invest Surg. 2017;30:125-132. 12. Ying KM, Chen Z, Dang CX, et al. Clinicopathology and Survival in Patients with Gastroesophageal Reflux After Radical Surgery of Proximal Gastric Cancer. Dig Dis Sci. 2018;63:1035-1042. 13. Mine S, Nunobe S, Watanabe M. A Novel Technique of Anti-reflux Esophagogastrostomy Following Left Thoracoabdominal Esophagectomy for Carcinoma of the Esophagogastric Junction. World J Surg. 2015;39:2359-2361. 14. Lee SS, Yu W, Chung HY, et al. Using Quality of Life Scales with Nutritional Relevance after Gastrectomy: a Challenge for Providing Personalized Treatment. J Gastric Cancer. 2017;17:342-353. 15. Tokunaga M, Hiki N, Ohyama S, et al. Effects of reconstruction methods on a patient's quality of life after a proximal gastrectomy: subjective symptoms evaluation using questionnaire survey. Langenbecks Arch Surg. 2009;394:637-641. 16. Analatos A, Lindblad M, Rouvelas I, et al. Evaluation of resection of the gastroesophageal junction and jejunal interposition (Merendino procedure) as a rescue procedure in patients with a failed redo antireflux procedure. A single-center experience. BMC Surg. 2018;18:70. 17. Nomura E, Lee SW, Kawai M, et al. Functional outcomes by reconstruction technique following laparoscopic proximal gastrectomy for gastric cancer: double tract versus jejunal interposition. World $\mathrm{J}$ Surg Oncol. 2014;12:20. 18. Huang Y, Wang S, Shi Y, et al. Uncut Roux-en-Y reconstruction after distal gastrectomy for gastric cancer. Expert Rev Gastroenterol Hepatol. 2016;10:1341-1347. 19. Park JY, Kim YJ. Uncut Roux-en-Y Reconstruction after Laparoscopic Distal Gastrectomy Can Be a Favorable Method in Terms of Gastritis, Bile Reflux, and Gastric Residue. J Gastric Cancer. 2014;14:229-237. 20. Kim SG, Ji SM, Lee NR, et al. Quality of Life after Endoscopic Submucosal Dissection for Early Gastric Cancer: A Prospective Multicenter Cohort Study. Gut Liver. 2017;11:87-92. 21. Woo A, Fu T, Popovic M, et al. Comparison of the EORTC STO-22 and the FACT-Ga quality of life questionnaires for patients with gastric cancer. Ann Palliat Med. 2016;5:13-21. 22. Heits N, Bernsmeier A, Reichert B, et al. Long-term quality of life after endovac-therapy in anastomotic leakages after esophagectomy. J Thorac Dis. 2018;10:228-240. 23. Huang Q, Zhong J, Yang T, et al. Impacts of anastomotic complications on the health-related quality of life after esophagectomy. J Surg Oncol. 2015;111:365-370. 24. Yang K, Bang HJ, Almadani ME, et al. Laparoscopic Proximal Gastrectomy with Double-Tract Reconstruction by Intracorporeal Anastomosis with Linear Staplers. J Am Coll Surg. 2016;222:e39-45. 25. Jung DH, Lee Y, Kim DW, et al. Laparoscopic proximal gastrectomy with double tract reconstruction is superior to laparoscopic total gastrectomy for proximal early gastric cancer. Surg Endosc. 2017;31:3961-3969. 26. Wang S, Lin S, Wang H, et al. Reconstruction methods after radical proximal gastrectomy: A systematic review. Medicine (Baltimore). 2018;97:e0121. 27. Isobe T, Hashimoto K, Kizaki J, et al. Reconstruction methods and complications in proximal gastrectomy for gastric cancer, and a comparison with total gastrectomy. Kurume Med J. 2014;61:23-29. 28. Nakamura M, Yamaue H. Reconstruction after proximal gastrectomy for gastric cancer in the upper third of the stomach: a review of the literature published from 2000 to 2014. Surg 
Today. 2016;46:517-527. 29. Kumagai K, Shimizu K, Yokoyama N, et al. Questionnaire survey regarding the current status and controversial issues concerning reconstruction after gastrectomy in Japan. Surg Today. 2012;42:411-418. 30. Sugoor P, Shah S, Dusane R, et al. Proximal gastrectomy versus total gastrectomy for proximal third gastric cancer: total gastrectomy is not always necessary. Langenbecks Arch Surg. 2016;401:687-697. 31. Wen L, Chen XZ, Wu B, et al. Total vs. proximal gastrectomy for proximal gastric cancer: a systematic review and meta-analysis. Hepatogastroenterology. 2012;59:633640. 32. Kim DJ, Kim W. Laparoscopy-assisted Proximal Gastrectomy with Double Tract Anastomosis Is Beneficial for Vitamin B12 and Iron Absorption. Anticancer Res. 2016;36:4753-4758. 33. Park JY, Park KB, $\mathrm{Kwon} \mathrm{OK}$, et al. Comparison of laparoscopic proximal gastrectomy with double-tract reconstruction and laparoscopic total gastrectomy in terms of nutritional status or quality of life in early gastric cancer patients. Eur J Surg Oncol. 2018;44:1963-1970. 34. Nomura E, Lee SW, Tokuhara T, et al. Functional outcomes according to the size of the gastric remnant and type of reconstruction following open and laparoscopic proximal gastrectomy for gastric cancer. Hepatogastroenterology. 2012;59:1677-1681. 35. Sakuramoto S, Yamashita K, Kikuchi S, et al. Clinical experience of laparoscopy-assisted proximal gastrectomy with Toupet-like partial fundoplication in early gastric cancer for preventing reflux esophagitis. J Am Coll Surg. 2009;209:344-351. 36. Ahn SH, Jung DH, Son SY, et al. Laparoscopic doubletract proximal gastrectomy for proximal early gastric cancer. Gastric Cancer. 2014;17:562-570. 37. Yoo $\mathrm{CH}$, Sohn $\mathrm{BH}, \mathrm{Han} \mathrm{WK}$, et al. Proximal gastrectomy reconstructed by jejunal pouch interposition for upper third gastric cancer: prospective randomized study. World J Surg. 2005;29:1592-1599. 38. Takagawa R, Kunisaki C, Kimura J, et al. A pilot study comparing jejunal pouch and jejunal interposition reconstruction after proximal gastrectomy. Dig Surg. 2010;27:502-508.

\section{Figure Legends}

Fig. 1 Proximal gastrectomy. A, B The right gastroepiploic artery was divided with preservation of the right gastroepiploic arcus vasculosi. C, D The gastric corpus was incised and closed vertically on the side of the greater curvature in the nonvascular area between the left and right gastroepiploic arteries using a single-use stapler. E, F The gastric corpus was incised and closed from the gastric stump to the proposed cutting site in the lesser curvature, and then the gastric tube was formed (d duodenum, g stomach, arrow right gastroepiploic artery).

Fig. 2 Roux-en-Y anastomosis plus occlusion of the antrum. A, B: A 3-cm incision was made in the anterior wall of the gastric body at the lowest site in the greater curvature. Then, a circular stapler was inserted into the residual stomach for side-end anastomosis of the esophagus and the stomach. C, D A 60-mm stapler was used to close the antrum at a site $2-3 \mathrm{~cm}$ distal to the pylorus through the space between the vascular arch and the curvature of the gastric antrum. E, F Occlusion of the antrum was completed. G, H The jejunum was divided at a site $15 \mathrm{~cm}$ distal to the ligament of Treitz; gastrojejunal side-side anastomosis was completed by sutures approximately $3-5 \mathrm{~cm}$ distal to the stump of the efferent loop (e abdominal esophagus, g stomach, $\mathrm{p}$ pylorus, $\mathrm{d}$ duodenum, j jejunum, arrow occlusion line, E-G esophagogastrostomy, G-J gastrojejunostomy). 
Fig. 3 A general view of the reconstructed digestive tract using the novel method. A, B The reconstructed digestive tract includes an esophageal-gastric-jejunal tract and a physiological-state gastric antrumduodenal tract with an occluded proximal antrum (e abdominal esophagus, g stomach, d duodenum, ro roux limb, af afferent loop, ef efferent loop, arrow uncut block line, E-G esophagogastrostomy, G-J gastrojejunostomy, J-J jejunojejunostomy).

\section{Figures}




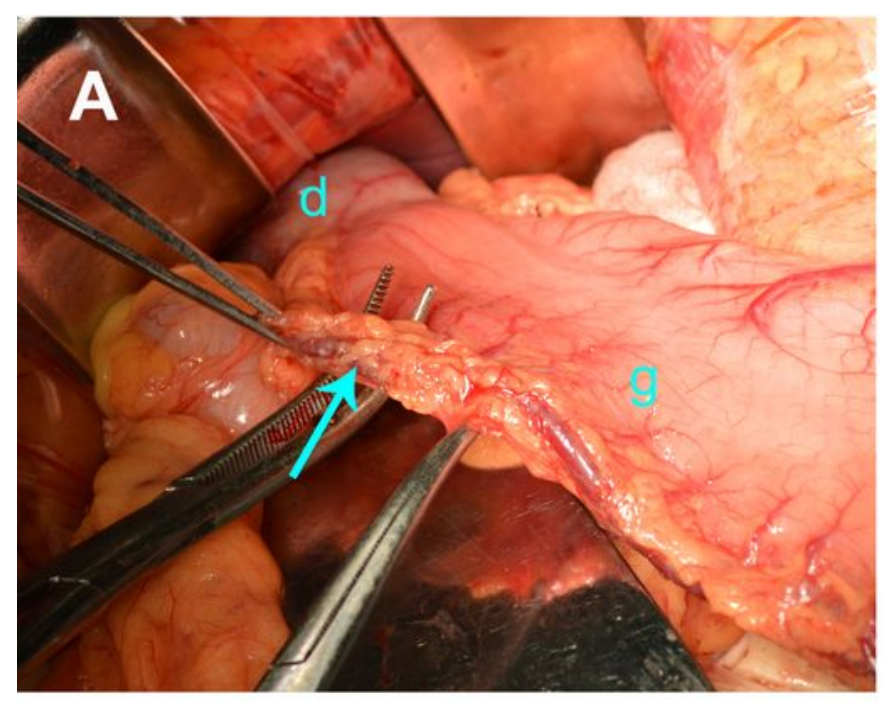

\section{B}
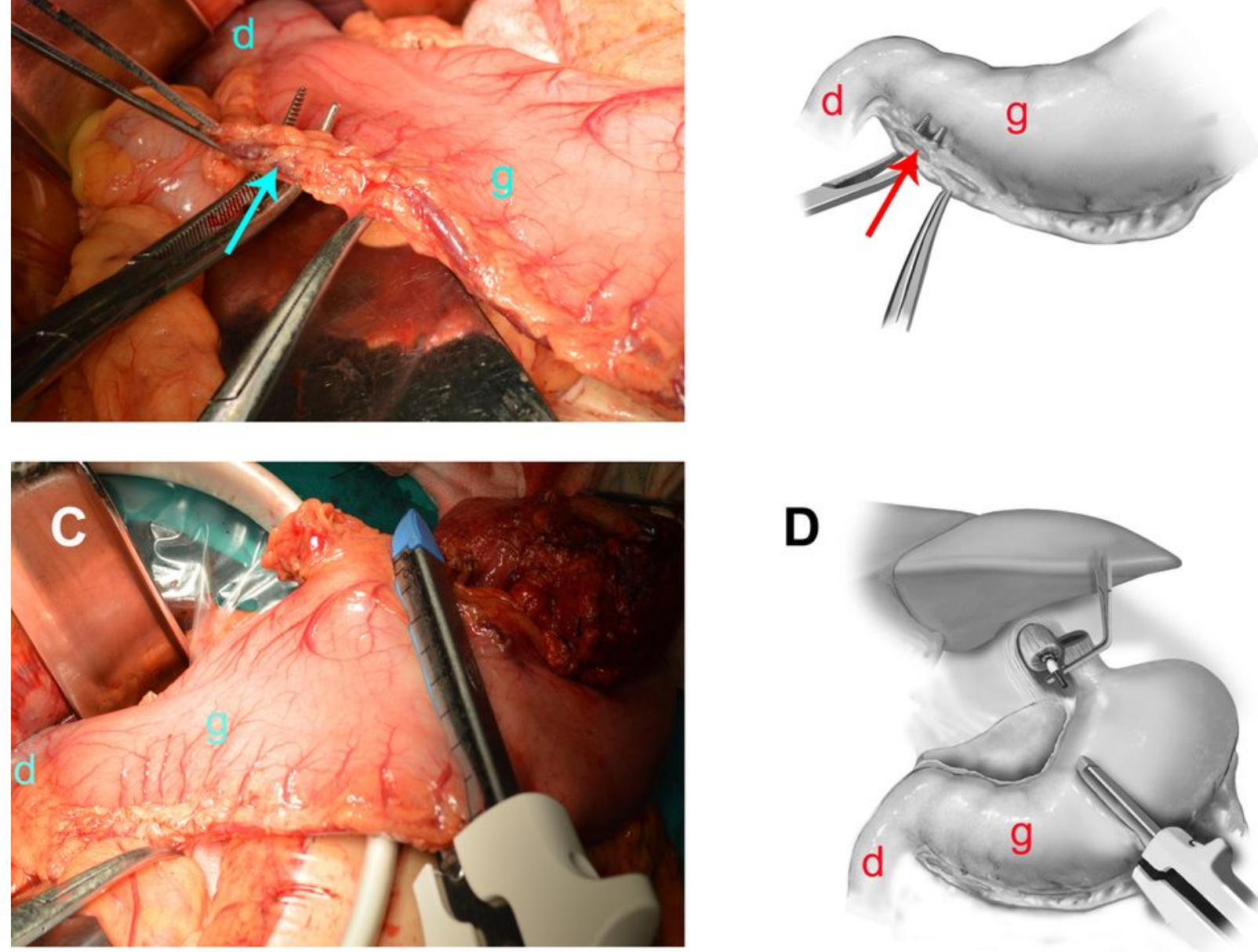

D
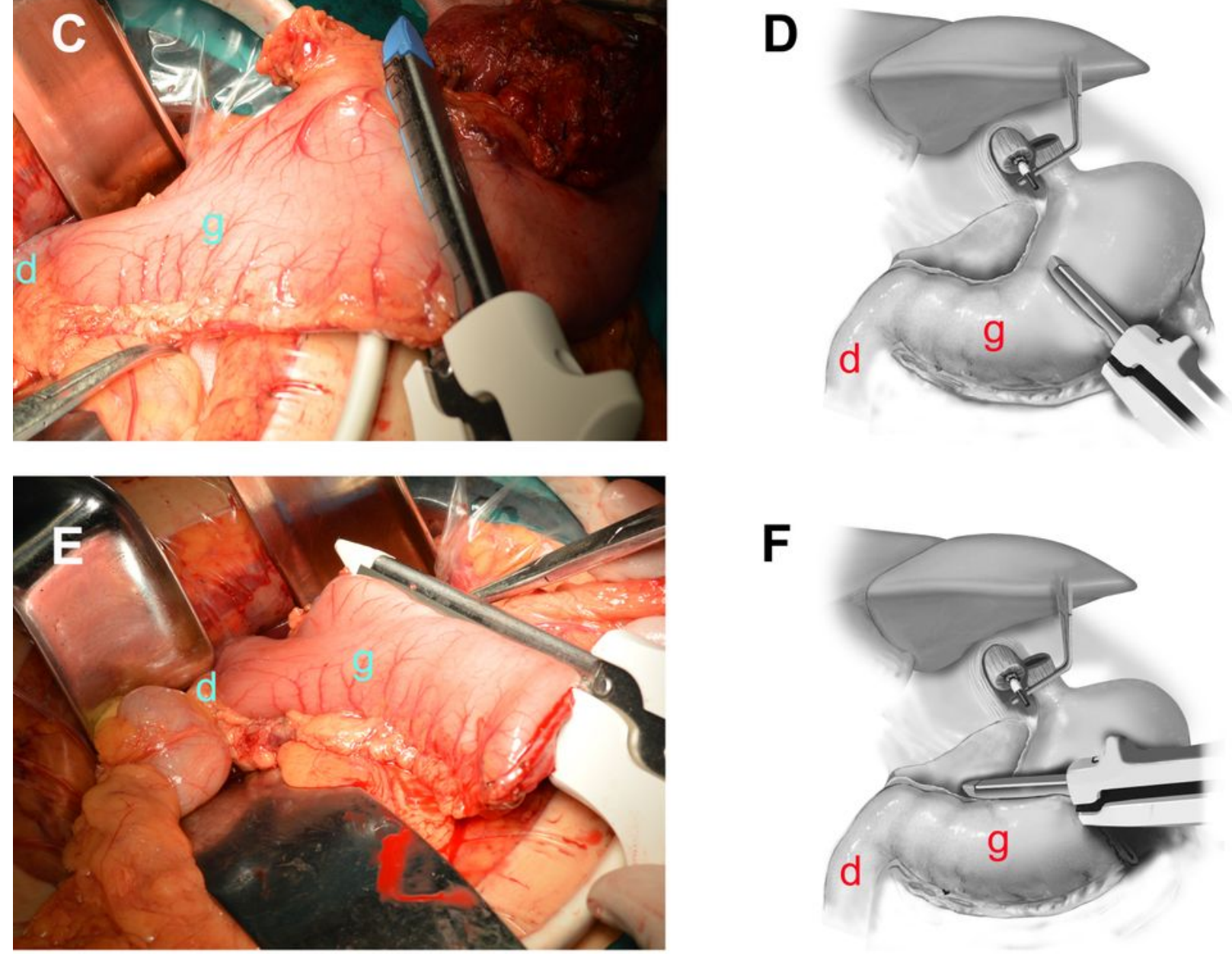

Figure 1

Proximal gastrectomy. A, B The right gastroepiploic artery was divided with preservation of the right gastroepiploic arcus vasculosi. C, D The gastric corpus was incised and closed vertically on the side of the greater curvature in the nonvascular area between the left and right gastroepiploic arteries using a single-use stapler. E, F The gastric corpus was incised and closed from the gastric stump to the proposed 
cutting site in the lesser curvature, and then the gastric tube was formed (d duodenum, $\mathrm{g}$ stomach, arrow right gastroepiploic artery).
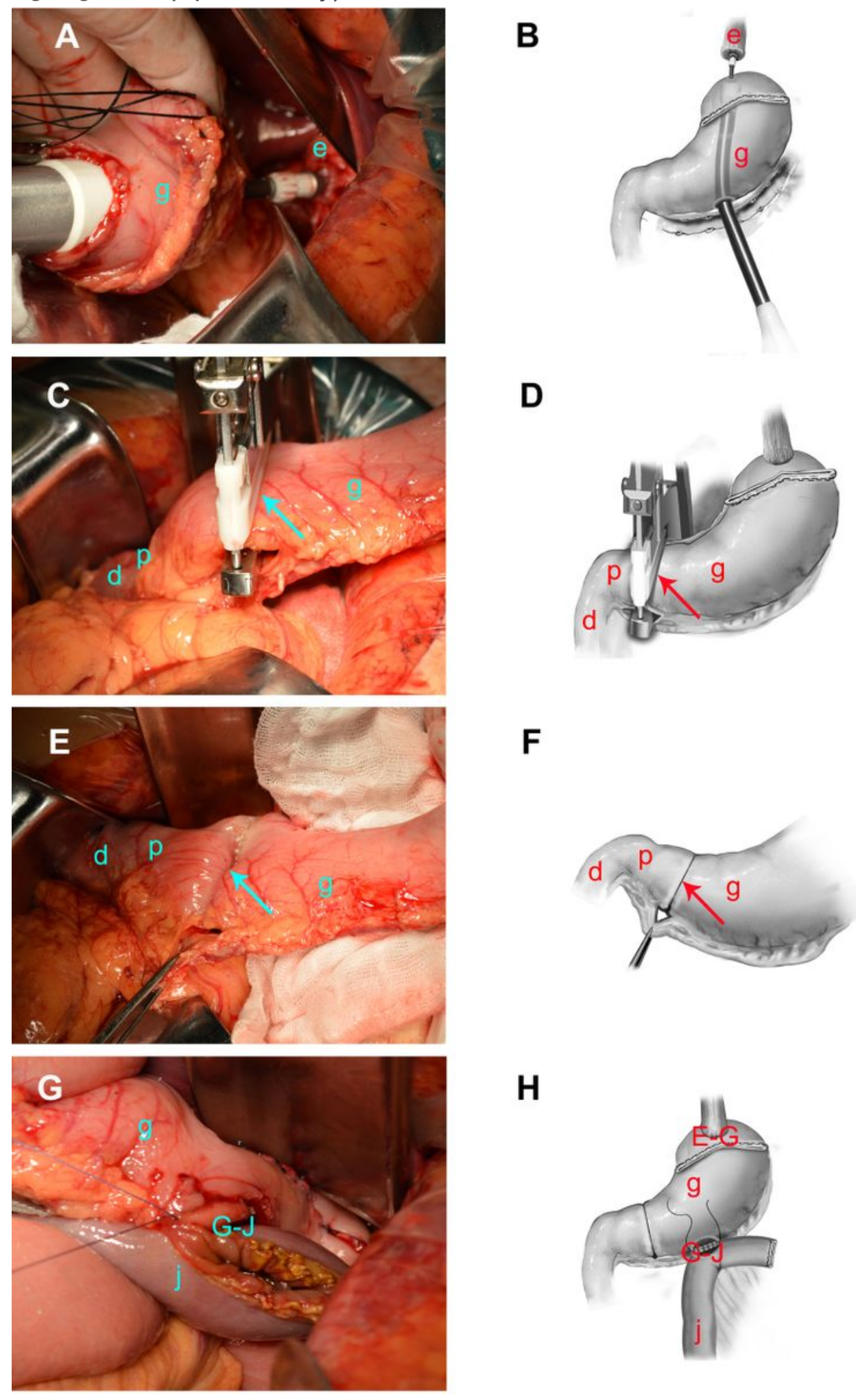

Figure 2

Roux-en-Y anastomosis plus occlusion of the antrum. A, B: A 3-cm incision was made in the anterior wall of the gastric body at the lowest site in the greater curvature. Then, a circular stapler was inserted into the residual stomach for side-end anastomosis of the esophagus and the stomach. C, D A 60-mm stapler 
was used to close the antrum at a site $2-3 \mathrm{~cm}$ distal to the pylorus through the space between the vascular arch and the curvature of the gastric antrum. E, F Occlusion of the antrum was completed. G, H The jejunum was divided at a site $15 \mathrm{~cm}$ distal to the ligament of Treitz; gastrojejunal side-side anastomosis was completed by sutures approximately $3-5 \mathrm{~cm}$ distal to the stump of the efferent loop (e abdominal esophagus, $g$ stomach, $p$ pylorus, d duodenum, j jejunum, arrow occlusion line, E-G esophagogastrostomy, G-J gastrojejunostomy).
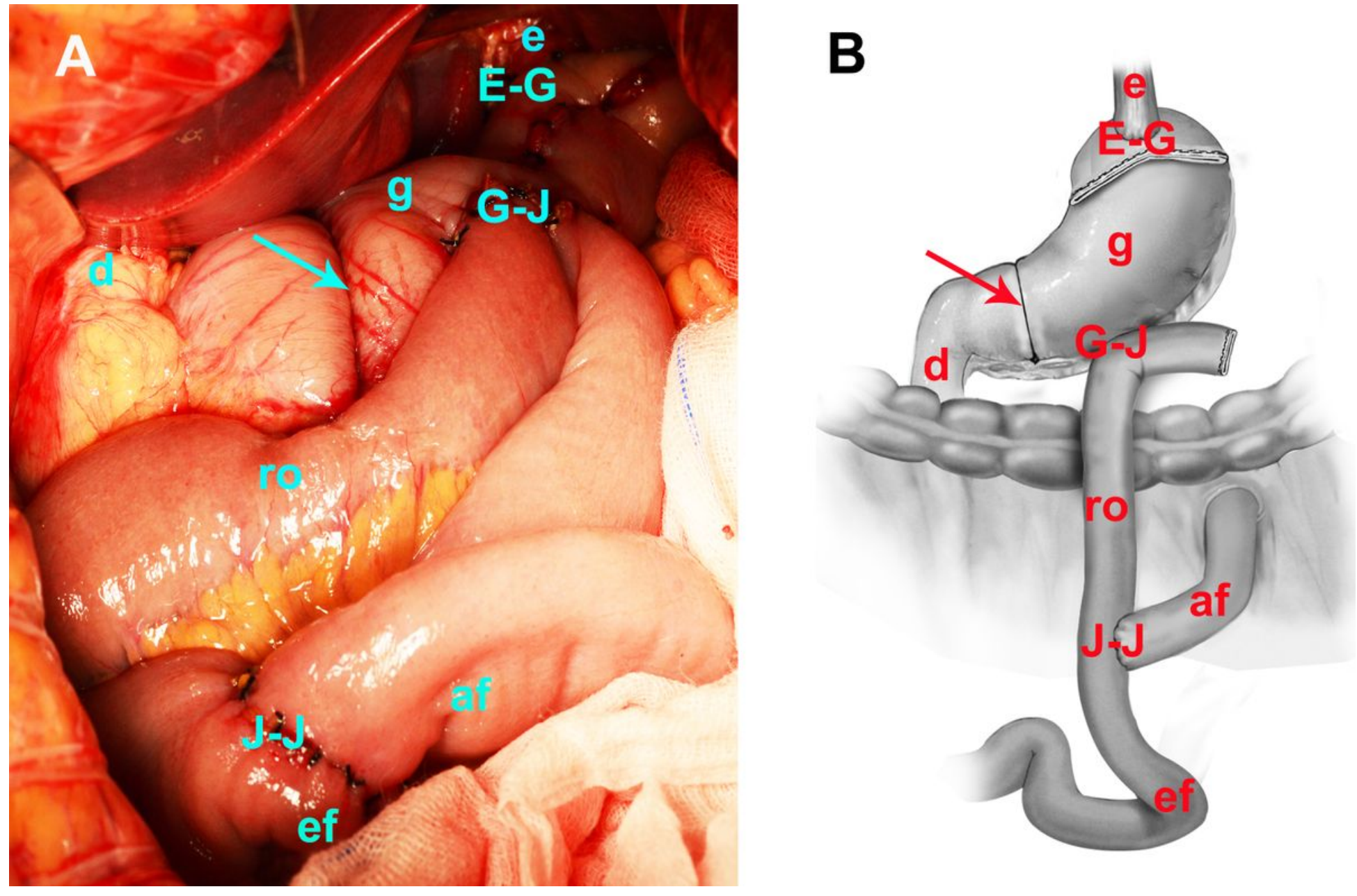

Figure 3

A general view of the reconstructed digestive tract using the novel method. A, B The reconstructed digestive tract includes an esophageal-gastric-jejunal tract and a physiological-state gastric antrumduodenal tract with an occluded proximal antrum (e abdominal esophagus, $g$ stomach, $d$ duodenum, ro roux limb, af afferent loop, ef efferent loop, arrow uncut block line, E-G esophagogastrostomy, G-J gastrojejunostomy, J-J jejunojejunostomy).

\section{Supplementary Files}

This is a list of supplementary files associated with this preprint. Click to download.

- supplement1.pdf 